\title{
Alcohol-related Liver Disease: Areas of Consensus, Unmet Needs and Opportunities for \\ Further Study
}

Gyongyi Szabo gyongyi.szabo@umassmed.edu*1\#, Patrick S. Kamath kamath.patrick@mayo.edu ${ }^{2 *}$, Vijay H. Shah shah.vijay@mayo.edu*3, Mark Thursz m.thursz@imperial.ac.uk**4,Philippe Mathurin philippe.mathurin@chru-lille.fr*5

\section{*All authors contributed equally to the work in this manuscript \#Corresponding Author}

${ }^{1}$ University of Massachusetts Medical School, Department of Medicine, 364 Plantation Street, LRB-208, Worcester, MA, USA 01605-2324

${ }^{2}$ Mayo Clinic, Division of GI and Internal Medicine, 200 First Street SW, Rochester, MN, USA 55905

${ }^{3}$ Mayo Clinic, Gastroenterology and Hepatology, 200 First Street, SW, Rochester, MN, USA 55905

${ }^{4}$ Imperial College of School of Medicine at St. Mary's, Department of Medicine, Norfolk Place, London, UK W21NY, 0207-594-3851

${ }^{5}$ Hôpital Huriez, Service des maladies de l'appareil digestif, Rue Polonovski, Lille, FR F-59037 Lille

See Appendix for the full list of all speakers from the Conference.

This report summarizes the highlights of the first AASLD-EASL Joint Conference on Alcoholrelated Liver Disease and Alcoholic Hepatitis.

Keywords: Alcoholic Liver Disease; Alcoholic Hepatitis 


$\begin{array}{ll}\text { Corresponding Author: } & \text { Gyongyi Szabo } \\ & \text { University of Massachusetts Medical School } \\ & \text { Department of Medicine, LRB 208 } \\ & \text { 364 Plantation Street } \\ & \text { Worcester, MA, USA 01605-2324 } \\ & \text { 508-856-5275 } \\ & \text { Gyongyi.Szabo@ umassmed.edu }\end{array}$

Abbreviations: European Association for the Study of the Liver (EASL); American Association for the Study of Liver Disease (AASLD); nonalcoholic fatty liver disease (NAFLD); nonalcoholic steatohepatitis (NASH); alcoholic hepatitis (AH); Maddrey Discriminant Function (MDF); magnetic resonance (MR); United Network for Organ Sharing (UNOS)

Financial Support: none 
A joint meeting of the European Association for the Study of the Liver (EASL) and the American Association for the Study of Liver Disease (AASLD) was held in London on September 30 and October 1, 2017. The goals of the meeting were to identify areas of broad agreement and disagreement, develop consensus, and inform future directions to ultimately reduce the burden, morbidity, and mortality of alcohol-related liver disease (previously termed alcoholic liver disease). The specific aims of the meeting were to identify unmet needs and areas for future investigation to reduce alcohol consumption; develop markers for diagnosis and prognosis of disease; and create a framework to test novel pharmacological agents with prespecified treatment end-points. A table summary of these goals and aims is provided in context of epidemiology (Table 1), current management strategies (Table 2), next steps for future trials (Table 3) and translational science (Table 4).

\section{EPIDEMIOLOGY, ADDICTION, DIAGNOSIS AND DISEASE PROGRESSION FACTORS IN ALCOHOL-RELATED LIVER DISEASE} Epidemiology

Worldwide, approximately 2.4 billion people consume alcohol with $1 \cdot 5$ billion $(1 \cdot 4-1 \cdot 6)$ male current drinkers and $0 \cdot 9$ billion $(0 \cdot 8-1 \cdot 0)$ female current drinkers (1). Approximately 2 million globally die of liver disease each year, and up to $50 \%$ of mortality with cirrhosis is attributable to alcohol. Alcohol-related liver disease represents one of the top 30 causes of death in recent studies of global burden of disease. In 2010, the worldwide rate of alcohol-attributable cirrhosis death was 7.2 deaths per 100,000 people (4.6 in females and 9.7 in males) (2).

Total per capita consumption of alcohol varies from continent to continent, from country to country within a continent, and from region to region within a country. As an example, total 
per capita annual consumption is at about $10 \mathrm{~L} /$ adult in the U.S., 12-13 L in France, 11-12 L in the UK, 7-8 L in Italy and 11-13 L in Eastern Europe, whereas it is only 0-2 L in North Africa/Middle East (3).

Regardless of the variation from country to country, liver-related death rates correlate with alcohol consumption in a given country. For example, a decrease in overall alcohol consumption in the wine-drinking countries of southern Europe has driven reduction in cirrhosis mortality rates whereas a substantial increase has been observed in Britain for both men and women (4). Despite the high global burden of mortality due to alcohol-related liver disease, both in terms of number of deaths and in terms of years of life lost, the estimates may actually underrepresent the true burden of disease. Because of this, international comparisons of the burden of alcohol-related liver disease are problematic and often unreliable. Indeed, much of this information relies on coding of death certificates. An example of coding errors includes a lack of willingness of many providers to attribute cirrhosis death to alcohol. This may lead to significant under-reporting of alcohol-related liver disease, especially alcohol-related cirrhosis. It is also unknown whether the risk of alcohol associated liver disease depends on the type of alcohol imbibed, whether alcohol is consumed with foods or on an empty stomach, whether certain foods are protective, and whether binge drinking clearly confers a higher risk. The severity of alcohol-related liver disease in all its stages is potentiated by obesity, but genetic risk factors for both alcohol misuse and alcohol-related liver disease are unclear.

\section{Public Health Policy relating to alcohol-related liver disease}

Given the link between alcohol consumption and alcohol-related liver disease, it follows that public health policy substantially influences mortality rates. In fact, public policies are more 
effective than education and approaches at the individual level. The dominant strategies for public intervention include pricing and marketing (5), with price being the single strongest driver of alcohol use. When pricing rises, alcohol consumption and alcohol-related liver disease commensurately decrease. Conversely, when prices drop, alcohol consumption and alcoholrelated death rates increase. The best approach for pricing strategies focuses on taxation. Availability can also be regulated at the government level by determining days and hours of sale and state control over outlets of sale. Simple measures to reduce alcohol consumption have been proposed, such as regular incremental above inflation tax increase, a minimal price of alcohol, protecting children from alcohol marketing, and clinicians, especially primary care physicians, advising all their patients to reduce alcohol consumption (5). Finally, government can also regulate a number of marketing features, including the prevention of targeting selected vulnerable populations such as young individuals and banning sports sponsorship. Despite these tools that government possesses to influence alcohol consumption, there are a number of counter-regulatory goals and lobbying initiatives that combat the effectiveness of government regulations.

\section{Alcohol Use Disorder}

Alcohol use disorders represent a chronic and relapsing disease which affects nearly one in ten individuals of the general population in the western world. Given that continued alcohol consumption after the onset of liver disease increases liver-related morbidity and mortality, the ideal focus of treatment in these patients is long-term abstinence and prevention of relapse. There is strong evidence that the most effective strategy to reduce alcohol intake, promote abstinence, and prevent relapse at the individual level is the combination of psychosocial and 
pharmacologic intervention (6). One of the few randomized controlled trials comparing modes of psychosocial therapy in patients specifically with alcohol related liver disease supported the use of motivational enhancement therapy although other counseling approaches are also widely utilized $(7,8)$. A number of medications have been approved for treatment of alcohol-use disorders including disulfiram, naltrexone, nalmefene, and acamprosate (9). Additional drugs are also under intense investigation, including topiramate, ondansetron, and baclofen (10). However, a major limitation is that most of these drugs have not been tested in patients with alcohol use disorder who also have advanced liver disease. The medication with the strongest evidence for effectiveness and safety in this group of patients is baclofen given that this is the only anti-craving medication formally tested in randomized controlled trials in patients with alcohol use disorder and cirrhosis (11). Baclofen is approved in some countries but is not FDA approved in the U.S. for this use. Thus, a major unmet need is further validation of a pharmacologic intervention for patients with alcohol use disorders with cirrhosis. Further complicating this issue is the lack of proper care models in many parts of the world (e.g., patients with alcohol use disorder and cirrhosis fall in the gap between addiction specialists and hepatologists). Another unmet need is a reliable and discrete remote monitoring and/or digital health solutions for ascertaining alcohol consumption. Nonetheless, screening for alcohol related liver disease in high risk populations is recommended at the primary care level. Based on recent evidence screening strategies have been proposed for alcohol related liver disease in at-risk populations although validation is required (12). 


\section{Co-factors and Genetics}

Progression and prognosis of alcohol-related liver disease is influenced by important co-

factors, most notably, fatty liver disease due to metabolic syndrome and viral hepatitis. Indeed, there has been a progressive rise in the prevalence of obesity and of Type II diabetes in the general population and alcohol-related liver disease is frequently superimposed on obesity. Recent population-based studies have found that obese individuals who consume alcohol may have a greater likelihood of having hepatic steatosis and alcohol consumption increases the risk of fatty liver in individuals with obesity. Furthermore, obesity may negatively impact alcoholrelated liver disease progression and prognosis. These observations suggest that there are additive and/or synergistic interactions of variables associated with nonalcoholic fatty liver disease (NAFLD) in combination with variables associated with alcohol-related liver disease, most notably the amount of alcohol consumption. This has led to recommendations to curtail alcohol use in individuals with nonalcoholic steatohepatitis (NASH) and conversely to manage weight and risk factors for NASH in individuals with alcohol-related liver disease. Though small studies have suggested there may be potential beneficial effects of low levels of alcohol consumption in individuals with NASH, the majority of studies showing a benefit of low levels of alcohol consumption are focused in healthy individuals. In a recent study, alcohol use $\geq 210 \mathrm{~g} /$ week for men and $\geq 140 \mathrm{~g} /$ week for women was associated with a higher risk of severe complications of liver disease in the general population (13). The interaction between alcohol consumption and obesity is clearly an area that needs further investigation. Another co-variable is non-hepatic co-morbidity that is increasing in patients with alcohol-related liver disease (14). Indeed, a recent analysis of electronic medical records demonstrate that the Charlson Comorbidity Index has increased substantially over the last decade and that patients in the U.S. 
presenting with alcoholic hepatitis $(\mathrm{AH})$ are older and more likely to have multiple systemic comorbidities such as COPD (14). This may also adversely affect the prognosis of alcoholrelated liver disease. However, there are positive co-factors to consider as well. For example, coffee drinking has been shown to be protective in alcohol-related liver disease although this requires much more detailed investigation before application can be proposed (15). There may also be microbiome characteristics that afford protection of more severe prognoses in patients with alcohol-related liver disease.

There is now increasing evidence that supports a genetic basis for alcohol-related liver disease. Since all individuals who drink in excess do not develop liver disease there is clearly a genetic risk in alcohol-related liver disease. The genetic loci that have been best studied and validated include PNPLA33, TM6SF2, and MBOAT7 $(16,17)$. It is important to note that these genetic profiles are distinct from other genetic loci, which may predispose towards alcohol misuse as aforementioned loci focus on liver injury in response to alcohol consumption. The allelic risk conferred by these genetic variants approximates about 2.5 -fold which is surprisingly high for a genetically-complex disorder such as alcohol-related liver disease and may be related to biases related to the choice of the control groups (16). Interestingly, all three of these mutations are also risk factors for NAFLD/NASH and all three genes are "lipid genes" (18). For example, PNPLA3 is a triglyceride lipase, while TM6SF2 plays a role in VLDL lipidation, and MBOAT7 is a lysophosphatidylinositol acyltransferase (18). It may be that PNPLA3 is most relevant for steatosis and hepatocellular cancer in alcohol-related liver disease, while TM6SF2 is relevant for inflammation and fibrosis progression in hepatocellular cancer, and MBOAT7 is most notable for fibrosis progression (16). However, this hypothesis needs further validation. Most recently, a splice variant encoding a lipid droplet protein was associated with a reduced risk 
of progression from steatosis to hepatitis (19). Future studies will hopefully allow us to "individualize" care for patients with alcohol-related liver disease based on genetic predispositions, especially if "druggable" targets can be developed for the phenotypic alterations associated with the genetic profiles.

\section{Alcohol-related Liver Disease}

Alcohol-related liver disease covers a spectrum including fatty liver disease, alcoholic hepatitis, and cirrhosis and its complications. The type and severity of steatosis and extent of fibrosis are independent predictive factors of fibrosis progression and the highest risk of disease progression is observed in heavy drinkers with alcoholic hepatitis (20-23). Approximately 3\% of patients with alcoholic hepatitis progress to cirrhosis annually. Epidemiological data show a strong correlation between severity and duration of alcohol abuse and the presence of cirrhosis. Among a cohort of 6970 adult subjects from a general population, the rate of cirrhosis was significantly higher in patients who consumed $\geq 30 \mathrm{~g} / \mathrm{d}$ than among abstinent controls or those with consumption $<30 \mathrm{~g} /$ day $(2.2 \%$ vs $0.08 \%)$. Subjects with alcohol consumption $>120 \mathrm{~g}$ /day had the highest risk of cirrhosis (around 13.5\% (24)).

Epidemiological data focusing on alcoholic hepatitis are sparse. In Denmark, from 1999 through 2008, the incidence of alcoholic hepatitis increased from 37 to 46 per million for men and from 24 to 34 per million for women. The increase in alcoholic hepatitis paralleled the increase in alcohol consumption (25). In the U.S., alcoholic hepatitis-related hospitalization increased from 249,884 in 2002 to 326,403 in 2010 (26). Cost of each hospitalization increased by $40.7 \%$ in 2010 compared to 2002 after adjustment on inflation over this period (26). Outside of these studies, information on burden of alcoholic hepatitis is missing and future 
epidemiological studies should focus on evolution of the burden of alcoholic hepatitis, including less severe disease.

The most dramatic presentation of alcohol-related liver disease is alcoholic hepatitis which manifests as rapid onset of jaundice and, in severe forms, may lead to acute-on-chronic liver failure with hepatic and extrahepatic organ failure, and mortality around $30 \%$ at one month. However, a symptomatic form of disease is also recognized where patients may be entirely asymptomatic but laboratory tests and liver biopsy show changes of steatohepatitis.

The currently accepted definition of alcoholic hepatitis is rapid onset of jaundice (bilirubin $>3 \mathrm{mg} / \mathrm{dL}$ ) with AST ( $>50 \mathrm{IU} / \mathrm{mL}$ ), and AST to ALT ratio of $>1.5$ in patients with heavy alcohol use (27). Heavy alcohol use is generally defined as more than 3 standard drinks per day for women (approximately 40 grams of alcohol), and four standard drinks per day for men (approximately 50-60 grams of alcohol). It is important to emphasize that standard drinks differ between countries. Liver biopsy remains the standard for making a diagnosis of alcoholic hepatitis though patients may be entered into clinical protocols with a clinical diagnosis, which is defined as heavy alcohol use with typical liver biochemistry and exclusion of other causes of liver disease. However, relying on clinical criteria alone may be associated with a risk of wrongly classifying patients with or without alcoholic hepatitis; on the other hand, there is also inter observer variation in assessment of severity of alcoholic hepatitis on liver biopsy.

In order to decrease the risk of misclassification for future studies evaluating drugs, the NIAAA consortium has proposed 3 groups (27): a) the most definitive diagnosis requires both clinical and histological documentation and has been referred to as definite alcoholic hepatitis (27); b) in the absence of histologic confirmation but typical liver biochemistry and exclusion of confounding variables, the diagnosis is referred to as probable alcoholic hepatitis (27); and c) in 
the absence of histology and in the presence of potential confounding variables, the diagnosis is possible alcoholic hepatitis (27).

Alcoholic hepatitis may be stratified as mild, moderate, and severe. Severe alcoholic hepatitis has traditionally been defined by a Maddrey Discriminant Function (MDF) score of $\geq 32$ that predicts mortality of up to $30 \%$ at 30 days (28-30). Other scorings such as the MELD score, ABIC score (28-30), and the Glasgow score (31) have also been used and may be superior to the MDF score (32). It is unclear whether change in any of these scores can be used as a surrogate end-point for survival, especially with less severe AH. Since patients with an MDF $\leq 32$ still have a significant risk for mortality, it has been proposed that the MELD score be used to stratify severity of alcoholic hepatitis. A MELD score $\geq 20$ is used to define severe alcoholic hepatitis which is associated with mortality being approximately $20 \%-40 \%$ at 90 days $(33,34)$. MELD score of 11 to 20 defines a group with moderately-severe alcoholic hepatitis; and MELD score $\leq$ 10 defines mild hepatitis. The natural history of the mild and moderately severe groups is unclear. It is also not known whether patients with moderately severe AH who do have a mortality risk have survival benefit from abstinence alone or if pharmacological therapy is required in addition.

The Lille score is used to determine response to steroid therapy at one week (35). Corticosteroids can be stopped at day 7 in non-responders (i.e., those with a Lille score $\geq 0.56$ ) as these patients do not benefit from continued treatment (36). It is unknown whether the Lille score is a valid marker of treatment outcome for therapies other than corticosteroids. A combination of MELD score at baseline and Lille score at 7 days may be used to assess mortality at two months and six months and seems to be more accurate, compared with either model alone (37). 
Invasive and Noninvasive Diagnosis of Alcohol-related Liver Disease

Biopsy is required to make a definitive diagnosis of $\mathrm{AH}$ (38-41), but use of liver biopsy as a standard of care for diagnosis varies throughout the world. Histology is also helpful for short-term prognosis of alcoholic hepatitis (41) and alcohol-related acute-on-chronic liver failure (42). For example, morphological evidence of bile accumulation (i.e., bilirubinostasis) is associated with the development of septic complications. Importantly, the degree of fibrosis is the main predictor of outcome in patients with compensated ALD (40). The characteristic histological findings of severe alcoholic hepatitis include macrovesicular steatosis, parenchymal inflammation with mononuclear cells and neutrophils, hepatocellular injury in the form of ballooning with abundant Mallory-Denk bodies, necrosis, and canalicular and/or ductular cholestasis. Hepatocellular ballooning is associated with deposition of collagen fibers resulting in pericellular and peri-sinusoidal fibrosis $(43,44)$. A majority of patients with symptomatic alcoholic hepatitis have underlying cirrhosis. When cirrhosis is established, histological features of alcoholic hepatitis and even pericellular fibrosis may not be prominent. While there is no "NAS score" equivalent for histology of alcoholic hepatitis as has been described for NASH, the histologic features are interchangeable in the two conditions in most cases. However, there is usually a greater disease severity with severe ballooning, necroinflammation and neutrophils surrounding hepatocytes (satellitosis) and bilirubinostasis in patients with alcoholic hepatitis as opposed to NASH (41). This may simply reflect the sicker nature of patients who undergo liver biopsy for alcoholic hepatitis as opposed to NASH. In addition, fibro-obliterative venous lesions and sclerosing hyaline necrosis are typical for alcohol associated liver injury and have not been described in NAFLD/NASH to date (43). 
Given the risks, cost, and inconvenience of invasive liver biopsy there is a large focus on non-invasive efforts to diagnose and determine prognosis in patients with alcohol-related liver disease. Non-invasive methods rely on two different but complementary approaches: serum biomarkers and the measurement of liver stiffness, using elastography modalities, either ultrasound- or magnetic resonance (MR)-based (45). Among ultrasound-based approaches, transient elastography, a relatively inexpensive and widely used point-of-care test, is the most validated for detection of advanced fibrosis, better at ruling out than ruling in the diagnosis of cirrhosis (46). However, there is no consensus on cut-offs for cirrhosis in the literature and there is a risk of false positive results in non-abstinent patients. Serum biomarkers have been also validated (47), but do not increase diagnostic performance when combined with transient elastography $(47,48)$. MR-based elastography is expensive, less available, and time consuming, but possibly more accurate for steatosis and fibrosis assessment (49). In general, unlike in patients with other etiologies of liver disease such as viral hepatitis, non-invasive methods are much less well validated in patients with alcohol-related liver diseases, especially in at-risk populations (12).

\section{Current Management and Treatment of Alcoholic Hepatitis}

Management of patients should be standardized in clinical trials for optimal assessment of the impact of novel agents. Lack of standardization may result in differences in outcome in clinical trials unrelated to the investigational agent. It has been difficult to stipulate that a liver biopsy be performed to confirm the diagnosis of alcoholic hepatitis prior to starting therapy. Biopsy could also potentially allow study of molecular pathways of hepatic injury that may 
inform future novel therapies. In the absence of liver biopsy, patients with "probable" alcoholic hepatitis may be treated as $\mathrm{AH}$ similar to those patients with biopsy-confirmed $\mathrm{AH}$.

There is broad agreement that management of severe alcoholic hepatitis requires treatment of the alcohol use disorder and risk for alcohol withdrawal, and treatment of the liver disease. Management of the liver disease in turn requires reversal of the alcoholic hepatitis and treatment of complications of alcohol-related cirrhosis including ascites, spontaneous bacterial peritonitis, hepatic encephalopathy, and variceal bleeding.

Treatment of alcoholic hepatitis includes nutritional supplementation to provide adequate protein and calories. Enteral supplementation is the preferred route because of safety and lower risk of infections. Patients require transfer to the Intensive Care Unit only if organ support is required. The role of routine antibiotic therapy is unclear and awaits the results of ongoing clinical trials. Since sepsis is difficult to diagnose in patients with AH, if sepsis is strongly suspected, and certainly when infection is diagnosed, broad-spectrum antibiotics should be initiated within 1 hour. The only pharmacological agent recommended for treatment of severe alcoholic hepatitis in the absence of contraindications is corticosteroids, namely prednisolone 40 $\mathrm{mg}$ daily or methylprednisolone, $32 \mathrm{mg}$ daily for 28 days. Pentoxifylline is not recommended as therapy for severe $\mathrm{AH}$.

Corticosteroids are associated with 1-month survival benefit in only about $60 \%$ of treated patients with severe alcoholic hepatitis and the benefit is not sustained intermediate or longerterm. Therefore, physicians may consider early liver transplantation in highly-selected patients with alcoholic hepatitis (50-52). The United Network for Organ Sharing (UNOS) (53), ACG Clinical Guideline (54) and the EASL clinical practice guidelines on ALD (45) suggest that listing a patient for transplantation should not be based only on the 6-month abstinence rule. 
When liver transplantation has been carried out in these highly-selected patients, survival has been excellent $(50-52,55)$. In terms of public opinion, a vast majority of the population of donors was not against early liver transplantation for alcoholic hepatitis (56). Early liver transplantation should be considered in the context of ethical principles recommending active treatment of patients, without discrimination, and according to best scientific knowledge (57). However, due to organ shortage and the stringency of the selection process limiting transplant availability, novel pharmacological agents to decrease mortality and progression of disease need to be developed for the majority of non-responders to medical therapy. Living-donor transplantation has been carried out in parts of the world where deceased-donor liver transplantation is unavailable. Such an approach requires discussion of the ethics of subjecting a donor to risk for an indication of liver transplantation that is still under investigation (57).

\section{Next Steps for Future Trials}

There are a number of reasons for the paucity of clinical trials in alcohol-associated liver disease. Recruitment to clinical trials in this group of patients can be challenging whilst endpoints such as mortality require large numbers of subjects to achieve meaningful levels of statistical power. Abstinence or recidivism inevitably affect patient outcomes but cannot be predicted. Nonetheless, clinical trials in early alcohol-related liver disease are very much needed to avoid the usual late presentation with decompensated liver disease. In order to achieve this end, patients could be selected in alcohol rehabilitation clinics. There is also evidence that universal screening for alcohol misuse in acute medical admissions is feasible and identifies patients at high risk of liver disease (58). Inclusion criteria should be defined and end-points may include prevention of liver-related mortality and complications of liver disease. 
Patients with alcoholic hepatitis are highly susceptible to infection and systemic inflammatory response syndrome which may be exacerbated by immunomodulatory drugs used to control hepatic inflammation. Finally, there is a lack of consensus around the design of clinical trials, particularly for the early phases of therapeutic development.

In alcoholic hepatitis there is a higher level of consensus around the type of patients who should be recruited into trials defined by inclusion and exclusion criteria. Patients with severe alcoholic hepatitis, defined by an $\mathrm{MDF} \geq 32, \mathrm{MELD} \geq 20$ or Glasgow alcoholic hepatitis score $\geq 9$, are considered a priority for therapeutic drug trials but it is now acknowledged that patients with milder disease states may also benefit from treatment given that the mortality in this group at 90 days may be as high as $15-20 \%$. Patients who have recently suffered a significant gastrointestinal hemorrhage where hemodynamic instability may have caused hepatic ischemia should be excluded. Similarly, patients with significant renal impairment (serum creatinine $>3$ $\mathrm{mg} / \mathrm{dl}$ ) at the time of randomization should be excluded as acute kidney injury is established as a poor prognostic factor. The need for ventilator or vasopressor support is also an exclusion criteria. Patients with viral hepatitis and active viral replication (HBsAg positive or HCV RNA positive) may be excluded as candidate immunomodulatory drugs might adversely affect the course of the viral infection. Active infection at the time of evaluation should not be considered an exclusion criterion, assuming the infection is controlled prior to randomization, as treated infection has been shown not to adversely influence mortality rates.

Recent progress in knowledge of the outcome of patients with $\mathrm{AH}$ allows experts to propose a more evidence-based approach that will help health agencies validate endpoints adapted to the specific stages of disease. Short-term outcome is mainly driven by severity of liver injury at baseline and early improvement in hepatic function whereas the most important 
determinant of outcome longer-term is abstinence (59). As a consequence, study designs testing therapeutic strategies that target the acute insult should focus on liver-related end-points for the short term. Three months seems to be the optimal end-point as $80 \%$ of the deaths at short-term occur within 3 months and relapse in alcoholism starting around 2-3 months does not significantly affect mortality at 3 months but is a contributing factor to long-term mortality. As a consequence, there is clear consensus that the primary outcome in phase III trials for patients with severe alcoholic hepatitis should be a mortality rate at 90 days. Future study designs may propose a 3-month duration of drug exposure in order to maintain improvement in liver injury over this period and avoid the potential bias of analysis of outcome at 3 months far removed from short treatment durations. It is also clear that pharmacological interventions may incur adverse events affecting mortality at earlier time points. Criteria need to be developed to ascertain DILI and drug-induced kidney injury in this population of patients who may have worsening of liver and kidney function as a result of the underlying disease alone. The number of patients required to show mortality benefit with a reasonable study power is high and new primary outcomes are required for phase II trials. Unfortunately, there is no consensus on the ideal surrogate primary endpoint. Changes in bilirubin levels at day 7 or the Lille score may be good indicators for some investigational agents but are unlikely to be appropriate for drugs with slower onset of activity or late side effects.

Only drugs that target either the key pathways involved in liver injury or the main mechanisms of early deaths demonstrated in clinical studies, translational research and animal models should be tested. Endpoints for phase I and II studies will be different from those proposed for phase III studies. An agreement of experts and health agencies is urgently required on the different primary end points for phase I, II and III studies, so that pharmaceutical 
companies and scientific societies can plan development of future clinical trials. Characteristics of patients included in phase I-II trials will also differ from those in phase III trials. The optimal candidates for phase I-II studies should be patients with low risk of mortality to ensure sufficient exposure to study drugs. Up to now, short-term mortality has been the only validated primary end point for testing drug efficacy in phase III studies. However, there is an urgent need to validate surrogate markers strongly associated with short-term mortality that may be used in the future as primary endpoints for phase III studies.

Unfortunately, there is no strong consensus on the treatment of patients randomized to the control arm of future studies. Survival at 28 days is improved by prednisolone but the drug has no beneficial effect beyond this point. Therefore, it makes sense to use prednisolone in the control arm in studies where the endpoint is 28-day survival and placebo when longer endpoints are being considered. Heterogeneity in the risk of adverse outcomes is widely recognized in patients with alcoholic hepatitis and stratification at the time of randomization might be usefully deployed in trials to minimize differences between active and control arms. Consensus is evolving towards using the MELD score for mortality stratification. Other potential stratification factors include treatment centers and risk of infection which might be estimated based on bacterial $16 \mathrm{~S}$ ribosomal DNA levels in whole blood samples.

A consistent reporting system should include the incidence of physician initiated courses of antibiotics (or antifungal agents), incidence of SIRS and the incidence of infection defined by the clinical criteria as recently proposed (60).

\section{Translational science and evolving biomarkers in alcohol-related liver disease}


New translational research approaches that cross science from the bench to bedside and from bedside to bench are promising. Basic science and clinical experts agree that translational science can aid the diagnosis and management of alcoholic hepatitis by identifying biomarkers and/or developing prediction models. There is a need for biomarkers that predict disease outcomes and response to therapy, early detection of infections, identify drug (steroid) resistance, or detect alcohol relapse.

Basic science observations from in vitro studies and from different animal models have greatly contributed to the increasing understanding of the pathogenetic mechanism of alcohol associated liver disease. However, there are limitations of the currently used animal models because none of them result in the full clinical spectrum of human acute alcoholic hepatitis. Animal models can be used to establish proof-of-concept with the caveat that different animal models may need to be used for different stages of alcohol-related liver disease. For example, the Lieber DeCarli chronic alcohol diet model results in fatty liver and very mild alcohol-induced liver injury (61). The acute-on-chronic alcohol administration in the NIAAA model shows some features of early alcoholic hepatitis including neutrophil infiltration and mild liver fibrosis (62). An acute binge in animals with chronic alcohol feeding increases liver damage and neutrophil infiltration (63). The continuous intragastric alcohol feeding model with weekly binge achieves most features of alcoholic hepatitis (64-66) including unique activation of the pyroptotic caspase 4/11-gasdermin-D pathway and its association with liver bacterial load as validated in severe alcoholic hepatitis patients but is expensive and requires special surgical expertise to develop (66). The salient features of human acute alcoholic hepatitis such as hyperbilirubinemia and fibrosis are not induced significantly in current murine models of alcohol-related liver disease. 
Combining a high fat diet with continued alcohol administration results in features of human alcoholic hepatitis; however, this models the combination of NASH and alcoholic hepatitis (67).

The evolution of alcohol-related liver disease into cirrhosis does not always include distinct episode(s) of alcoholic hepatitis. Little is known about early alcoholic hepatitis when liver inflammation is ongoing, but the patient is asymptomatic. Since these patients may eventually develop severe disease, clinical and translational studies should also focus on low grade alcoholic hepatitis to better understand the natural history of disease and impact a larger, currently neglected patient population.

Biomarker discovery is hampered by the lack of well-defined stages of alcohol-related liver disease. It remains to be determined whether liver biopsy is therefore required in clinical studies of biomarker discovery. Some argue that clinical parameters and/or MELD scores are sufficient for identification of biomarkers for disease progression and response to therapy. Additional consideration is related to selection of controls and references for biomarkers. Biomarker studies will likely need control groups including patients with alcohol use disorder without liver disease, patients with liver disease or cirrhosis due to factors other than alcohol, and normal healthy controls. Biomarker discovery and translational research may not only help in understanding pathogenesis of disease but may also be incorporated into clinical prediction models.

There are many candidate biomarkers that have been identified in discovery phases and await validation in large well-defined patient populations. Some of these include circulating indicators of gut microbial translocation (endotoxin, bacterial DNA), markers of systemic inflammation (cytokines, chemokines), sterile danger molecules (ST2, HMGB1), markers of apoptosis and cell death (fragments of keratin 18) and fibrosis markers (68-72) including 
cytochrome P4502E1. In addition to blood-based biomarkers, there are new potential

biomarkers in exhaled breath and urine. Finally, the composition of the microbiome in the gut and oral cavity may also represent useful sources of biomarkers in ALD.

\section{References}

1. Collaborators GBDA. Alcohol use and burden for 195 countries and territories, 1990-2016: a systematic analysis for the Global Burden of Disease Study 2016. Lancet 2018;392:1015-1035.

2. Rehm J, Samokhvalov AV, Shield KD. Global burden of alcoholic liver diseases. J Hepatol 2013;59:160-168.

3. Organization. WHOWH. Global status report on alcohol and health, 2014.; 2014.

4. Leon DA, McCambridge J. Liver cirrhosis mortality rates in Britain from 1950 to 2002: an analysis of routine data. Lancet 2006;367:52-56.

5. Sheron N. Alcohol and liver disease in Europe--Simple measures have the potential to prevent tens of thousands of premature deaths. J Hepatol 2016;64:957-967.

6. Addolorato G, Mirijello A, Barrio P, Gual A. Treatment of alcohol use disorders in patients with alcoholic liver disease. J Hepatol 2016;65:618-630.

7. Weinrieb RM, Van Horn DH, Lynch KG, Lucey MR. A randomized, controlled study of treatment for alcohol dependence in patients awaiting liver transplantation. Liver Transpl 2011;17:539-547.

8. Mathurin P, Ehrhard F. Management of alcohol dependence in transplant candidates: we are far away from the objective line. Liver Transpl 2011;17:492-493.

9. Vuittonet $\mathrm{CL}$, Halse M, Leggio L, Fricchione SB, Brickley M, Haass-Koffler CL, Tavares T, et al. Pharmacotherapy for alcoholic patients with alcoholic liver disease. Am J Health Syst Pharm 2014;71:1265-1276.

10. Addolorato G, Mirijello A, Leggio L, Ferrulli A, Landolfi R. Management of alcohol dependence in patients with liver disease. CNS Drugs 2013;27:287-299.

11. Addolorato G, Leggio L, Ferrulli A, Cardone S, Vonghia L, Mirijello A, Abenavoli L, et al. Effectiveness and safety of baclofen for maintenance of alcohol abstinence in alcohol-dependent patients with liver cirrhosis: randomised, double-blind controlled study. Lancet 2007;370:1915-1922. 12. Liangpunsakul S, Crabb DW. Early Detection of Alcoholic Liver Disease: Are We a Step Closer? Gastroenterology 2016;150:29-31.

13. Aberg F, Helenius-Hietala J, Puukka P, Farkkila M, Jula A. Interaction between alcohol consumption and metabolic syndrome in predicting severe liver disease in the general population. Hepatology 2018;67:2141-2149.

14. Nguyen TA, DeShazo JP, Thacker LR, Puri P, Sanyal AJ. The Worsening Profile of Alcoholic Hepatitis in the United States. Alcohol Clin Exp Res 2016;40:1295-1303.

15. Lourens S, Sunjaya D, Singal A, Liangpunsakul S, Puri P, Sanyal A, Ren X, et al. Acute Alcoholic Hepatitis: Natural History and Predictors of Mortality Using a Multicenter Prospective Study. Mayo Clinic Proceedings: Innovations, Quality \& Outcomes 2017;1:37-48.

16. Stickel F, Moreno C, Hampe J, Morgan MY. The genetics of alcohol dependence and alcoholrelated liver disease. J Hepatol 2017;66:195-211. 
17. Buch S, Stickel F, Trepo E, Way M, Herrmann A, Nischalke HD, Brosch M, et al. A genome-wide association study confirms PNPLA3 and identifies TM6SF2 and MBOAT7 as risk loci for alcohol-related cirrhosis. Nat Genet 2015;47:1443-1448.

18. Eslam M, Valenti L, Romeo S. Genetics and epigenetics of NAFLD and NASH: Clinical impact. J Hepatol 2018;68:268-279.

19. Abul-Husn NS, Cheng X, Li AH, Xin Y, Schurmann C, Stevis P, Liu Y, et al. A Protein-Truncating HSD17B13 Variant and Protection from Chronic Liver Disease. N Engl J Med 2018;378:1096-1106.

20. Galambos JT, Shapira R. Natural history of alcoholic hepatitis. IV. Glycosaminoglycuronans and collagen in the hepatic connective tissue. J Clin Invest 1973;52:2952-2962.

21. Marbet UA, Bianchi L, Meury U, Stalder GA. Long-term histological evaluation of the natural history and prognostic factors of alcoholic liver disease. J Hepatol 1987;4:364-372.

22. Mathurin P, Beuzin F, Louvet A, Carrie-Ganne N, Balian A, Trinchet JC, Dalsoglio D, et al. Fibrosis progression occurs in a subgroup of heavy drinkers with typical histological features. Aliment Pharmacol Ther 2007;25:1047-1054.

23. Pares A, Caballeria J, Bruguera M, Torres M, Rodes J. Histological course of alcoholic hepatitis. Influence of abstinence, sex and extent of hepatic damage. J Hepatol 1986;2:33-42.

24. Bellentani S, Saccoccio G, Costa G, Tiribelli C, Manenti F, Sodde M, Saveria Croce L, et al. Drinking habits as cofactors of risk for alcohol induced liver damage. The Dionysos Study Group. Gut 1997;41:845-850.

25. Sandahl TD, Jepsen P, Thomsen KL, Vilstrup H. Incidence and mortality of alcoholic hepatitis in Denmark 1999-2008: a nationwide population based cohort study. J Hepatol 2011;54:760-764.

26. Jinjuvadia R, Liangpunsakul S. Trends in Alcoholic Hepatitis-related Hospitalizations, Financial Burden, and Mortality in the United States. J Clin Gastroenterol 2015;49:506-511.

27. Crabb DW, Bataller R, Chalasani NP, Kamath PS, Lucey M, Mathurin P, McClain C, et al. Standard Definitions and Common Data Elements for Clinical Trials in Patients With Alcoholic Hepatitis:

Recommendation From the NIAAA Alcoholic Hepatitis Consortia. Gastroenterology 2016;150:785-790.

28. Carithers RL, Jr., Herlong HF, Diehl AM, Shaw EW, Combes B, Fallon HJ, Maddrey WC.

Methylprednisolone therapy in patients with severe alcoholic hepatitis. A randomized multicenter trial. Ann Intern Med 1989;110:685-690.

29. Mathurin P, Mendenhall CL, Carithers RL, Jr., Ramond MJ, Maddrey WC, Garstide P, Rueff B, et al. Corticosteroids improve short-term survival in patients with severe alcoholic hepatitis (AH): individual data analysis of the last three randomized placebo controlled double blind trials of corticosteroids in severe AH. J Hepatol 2002;36:480-487.

30. Ramond MJ, Poynard T, Rueff B, Mathurin P, Theodore C, Chaput JC, Benhamou JP. A randomized trial of prednisolone in patients with severe alcoholic hepatitis. N Engl J Med 1992;326:507512.

31. Forrest EH, Evans CD, Stewart S, Phillips M, Oo YH, McAvoy NC, Fisher NC, et al. Analysis of factors predictive of mortality in alcoholic hepatitis and derivation and validation of the Glasgow alcoholic hepatitis score. Gut 2005;54:1174-1179.

32. Forrest EH, Atkinson SR, Richardson P, Masson S, Ryder S, Thursz MR, Allison M, et al. Application of prognostic scores in the STOPAH trial: Discriminant function is no longer the optimal scoring system in alcoholic hepatitis. J Hepatol 2018;68:511-518.

33. Dunn W, Jamil LH, Brown LS, Wiesner RH, Kim WR, Menon KV, Malinchoc M, et al. MELD accurately predicts mortality in patients with alcoholic hepatitis. Hepatology 2005;41:353-358.

34. Kamath PS, Wiesner RH, Malinchoc M, Kremers W, Therneau TM, Kosberg CL, D'Amico G, et al. A model to predict survival in patients with end-stage liver disease. Hepatology 2001;33:464-470. 
35. Louvet A, Naveau S, Abdelnour M, Ramond MJ, Diaz E, Fartoux L, Dharancy S, et al. The Lille model: a new tool for therapeutic strategy in patients with severe alcoholic hepatitis treated with steroids. Hepatology 2007;45:1348-1354.

36. Mathurin P, O'Grady J, Carithers RL, Phillips M, Louvet A, Mendenhall CL, Ramond MJ, et al. Corticosteroids improve short-term survival in patients with severe alcoholic hepatitis: meta-analysis of individual patient data. Gut 2011;60:255-260.

37. Louvet A, Labreuche J, Artru F, Boursier J, Kim DJ, O'Grady J, Trepo E, et al. Combining Data From Liver Disease Scoring Systems Better Predicts Outcomes of Patients With Alcoholic Hepatitis. Gastroenterology 2015;149:398-406 e398; quiz e316-397.

38. Lucey MR, Mathurin P, Morgan TR. Alcoholic hepatitis. N Engl J Med 2009;360:2758-2769.

39. Mookerjee RP, Lackner C, Stauber R, Stadlbauer V, Deheragoda M, Aigelsreiter A, Jalan R. The role of liver biopsy in the diagnosis and prognosis of patients with acute deterioration of alcoholic cirrhosis. J Hepatol 2011;55:1103-1111.

40. Lackner C, Spindelboeck W, Haybaeck J, Douschan P, Rainer F, Terracciano L, Haas J, et al. Histological parameters and alcohol abstinence determine long-term prognosis in patients with alcoholic liver disease. J Hepatol 2017;66:610-618.

41. Altamirano J, Miquel R, Katoonizadeh A, Abraldes JG, Duarte-Rojo A, Louvet A, Augustin S, et al. $A$ histologic scoring system for prognosis of patients with alcoholic hepatitis. Gastroenterology 2014;146:1231-1239 e1231-1236.

42. Katoonizadeh A, Laleman W, Verslype C, Wilmer A, Maleux G, Roskams T, Nevens F. Early features of acute-on-chronic alcoholic liver failure: a prospective cohort study. Gut 2010;59:1561-1569.

43. Tiniakos DG, Anstee QM, Burt AD. Macsween's Pathology of the Liver (Seventh Edition): Elsevier, 2018.

44. Rangwala F, Guy CD, Lu J, Suzuki A, Burchette JL, Abdelmalek MF, Chen W, et al. Increased production of sonic hedgehog by ballooned hepatocytes. J Pathol 2011;224:401-410.

45. European Association for the Study of the Liver. Electronic address eee, European Association for the Study of the L. EASL Clinical Practice Guidelines: Management of alcohol-related liver disease. J Hepatol 2018;69:154-181.

46. Thiele M, Detlefsen S, Sevelsted Moller L, Madsen BS, Fuglsang Hansen J, Fialla AD, Trebicka J, et al. Transient and 2-Dimensional Shear-Wave Elastography Provide Comparable Assessment of Alcoholic Liver Fibrosis and Cirrhosis. Gastroenterology 2016;150:123-133.

47. Thiele M, Madsen BS, Hansen JF, Detlefsen S, Antonsen S, Krag A. Accuracy of the Enhanced Liver Fibrosis Test vs FibroTest, Elastography, and Indirect Markers in Detection of Advanced Fibrosis in Patients With Alcoholic Liver Disease. Gastroenterology 2018;154:1369-1379.

48. Voican CS, Louvet A, Trabut JB, Njike-Nakseu M, Dharancy S, Sanchez A, Corouge M, et al. Transient elastography alone and in combination with FibroTest $((R))$ for the diagnosis of hepatic fibrosis in alcoholic liver disease. Liver Int 2017;37:1697-1705.

49. Tapper EB, Loomba R. Noninvasive imaging biomarker assessment of liver fibrosis by elastography in NAFLD. Nat Rev Gastroenterol Hepatol 2018;15:274-282.

50. Im GY, Kim-Schluger L, Shenoy A, Schubert E, Goel A, Friedman SL, Florman S, et al. Early Liver Transplantation for Severe Alcoholic Hepatitis in the United States--A Single-Center Experience. Am J Transplant 2016;16:841-849.

51. Lee BP, Chen PH, Haugen C, Hernaez R, Gurakar A, Philosophe B, Dagher N, et al. Three-year Results of a Pilot Program in Early Liver Transplantation for Severe Alcoholic Hepatitis. Ann Surg 2017;265:20-29.

52. Mathurin P, Moreno C, Samuel D, Dumortier J, Salleron J, Durand F, Castel H, et al. Early liver transplantation for severe alcoholic hepatitis. N Engl J Med 2011;365:1790-1800. 
53. Beresford TP, Everson GT. Liver transplantation for alcoholic liver disease: bias, beliefs, 6-month rule, and relapse--but where are the data? Liver Transpl 2000;6:777-778.

54. Singal AK, Bataller R, Ahn J, Kamath PS, Shah VH. ACG Clinical Guideline: Alcoholic Liver Disease. Am J Gastroenterol 2018.

55. Addolorato G, Bataller R, Burra P, DiMartini A, Graziadei I, Lucey MR, Mathurin P, et al. Liver Transplantation for Alcoholic Liver Disease. Transplantation 2016;100:981-987.

56. Stroh G, Rosell T, Dong F, Forster J. Early liver transplantation for patients with acute alcoholic hepatitis: public views and the effects on organ donation. Am J Transplant 2015;15:1598-1604.

57. Donckier V, Lucidi V, Gustot T, Moreno C. Ethical considerations regarding early liver transplantation in patients with severe alcoholic hepatitis not responding to medical therapy. J Hepatol 2014;60:866-871.

58. Westwood G, Meredith P, Atkins S, Greengross P, Schmidt PE, Aspinall RJ. Universal screening for alcohol misuse in acute medical admissions is feasible and identifies patients at high risk of liver disease. J Hepatol 2017;67:559-567.

59. Louvet A, Labreuche J, Artru F, Bouthors A, Rolland B, Saffers P, Lollivier J, et al. Main drivers of outcome differ between short term and long term in severe alcoholic hepatitis: A prospective study. Hepatology 2017;66:1464-1473.

60. Bajaj JS, O'Leary JG, Reddy KR, Wong F, Olson JC, Subramanian RM, Brown G, et al. Second infections independently increase mortality in hospitalized patients with cirrhosis: the North American consortium for the study of end-stage liver disease (NACSELD) experience. Hepatology 2012;56:23282335.

61. Gao B, Xu MJ, Bertola A, Wang H, Zhou Z, Liangpunsakul S. Animal Models of Alcoholic Liver Disease: Pathogenesis and Clinical Relevance. Gene Expr 2017;17:173-186.

62. Bertola A, Mathews S, Ki SH, Wang H, Gao B. Mouse model of chronic and binge ethanol feeding (the NIAAA model). Nat Protoc 2013;8:627-637.

63. Ghosh Dastidar S, Warner JB, Warner DR, McClain CJ, Kirpich IA. Rodent Models of Alcoholic Liver Disease: Role of Binge Ethanol Administration. Biomolecules 2018;8.

64. Arteel GE. Animal models of alcoholic liver disease. Dig Dis 2010;28:729-736.

65. Tsukamoto H, Mkrtchyan H, Dynnyk A. Intragastric ethanol infusion model in rodents. Methods Mol Biol 2008;447:33-48.

66. Tsukamoto H, French SW, Benson N, Delgado G, Rao GA, Larkin EC, Largman C. Severe and progressive steatosis and focal necrosis in rat liver induced by continuous intragastric infusion of ethanol and low fat diet. Hepatology 1985;5:224-232.

67. Mathews S, Xu M, Wang H, Bertola A, Gao B. Animals models of gastrointestinal and liver diseases. Animal models of alcohol-induced liver disease: pathophysiology, translational relevance, and challenges. Am J Physiol Gastrointest Liver Physiol 2014;306:G819-823.

68. Bissonnette J, Altamirano J, Devue C, Roux O, Payance A, Lebrec D, Bedossa P, et al. A prospective study of the utility of plasma biomarkers to diagnose alcoholic hepatitis. Hepatology 2017;66:555-563.

69. Gonzalez-Quintela A, Garcia J, Campos J, Perez LF, Alende MR, Otero E, Abdulkader I, et al. Serum cytokeratins in alcoholic liver disease: contrasting levels of cytokeratin-18 and cytokeratin-19. Alcohol 2006;38:45-49.

70. Laursen TL, Stoy S, Deleuran B, Vilstrup H, Gronbaek H, Sandahl TD. The damage-associated molecular pattern HMGB1 is elevated in human alcoholic hepatitis, but does not seem to be a primary driver of inflammation. Apmis 2016;124:741-747.

71. Szabo G, Petrasek J. Gut-liver axis and sterile signals in the development of alcoholic liver disease. Alcohol Alcohol 2017;52:414-424. 
72. Ciocan D, Rebours V, Voican CS, Wrzosek L, Puchois V, Cassard AM, Perlemuter G.

Characterization of intestinal microbiota in alcoholic patients with and without alcoholic hepatitis or chronic alcoholic pancreatitis. Sci Rep 2018;8:4822. 


\section{Appendix}

EASL-AASLD Joint Meeting: Definition, therapeutic advances and clinical endpoints in alcoholic liver disease and alcoholic hepatitis.

London, United Kingdom - September 30 - October 1, 2017

Conference Speakers (in alphabetical order):

Giovanni Addolorato

Ramon Bataller

Patrizia Burra

Laurent Castera

Helena Cortez Pinto

Anna Mae Diehl

Bin Gao

Sir Ian Gilmore

Jochen Hampe

Rehm Jürgen

Patrick S. Kamath

Michael Karin

Alexander Krag

David Leon

Christopher Leptak

Alexandre Louvet

Michael Lucey

Philippe Mathurin

Craig McClain

Laura Nagy

Georges-Philippe Pageaux

Arun Sanyal

Bernd Schnabl

Vijay H. Shah

Gyongyi Szabo

Mark Thursz

Dina Tiniakos

Christian Trautwein

Hidekazu Tsukamoto 


\section{Table 1. Epidemiology, Addiction, Diagnosis, and Disease Progression Factors}

\section{Areas of Consensus}

Public policy: Price availability and marketing are best tactics to manage alcohol consumption at society level. Government policies are necessary to regulate marketing promotions via sports sponsorship, use of internet and social media, specifically those promotions that target vulnerable populations such as young individuals. Increasing consumption of alcohol amongst younger women is recognized as a public health concern as this population is at increased risk of development of alcohol-related liver disease. It is essential that this population be an important focus of alcohol reduction measures.

Health policies aiming to reduce per capita consumption should be emphasized to reduce the burden of alcohol related liver disease.

As with diabetes, national health policies should not only consider primary intervention to decrease alcohol use but should integrate in their plan secondary or tertiary interventions aiming to prevent development of alcohol associated liver disease and its complications in patients with alcohol use disorder.

Heavy alcohol with obesity leads to adverse consequences.

Alcohol rehabilitation should begin in hospital for AH patients while addiction trials should activate at time of discharge.

Surveillance for advanced hepatic fibrosis or cirrhosis with non-invasive methods should be promoted in patients with excessive alcohol consumption.

Policies are not uniform regarding early liver transplantation for patients with severe alcoholic hepatitis not responding to medical therapy. Societies need to establish guidelines for liver transplantation for alcoholic hepatitis based on local legislation framework.

\section{Unmet Needs and Opportunities for Future Study}

Pharmacologic therapy for AUD/ in patients with alcohol associated liver disease.

Define parameters for non-invasive imaging of the liver in patients with AUD.

Define subsets, predictors of outcome in patients with combined AH/NASH.

Epidemiological studies on the burden of alcoholic hepatitis

Which team member should primarily manage the patient with AUD/ALD.

Ideal endpoints for AUD/ALD addiction trials.

Non-invasive staging approach in AUD/ALD patients.

Best serologic nomogram for determining AH disease severity (MDF, MELD, etc.).

Role of liver biopsy in $\mathrm{AH}$. 
Safe level of alcohol consumption in patients with NASH. 


\section{Table 2. Current Management and Treatment of Alcoholic Hepatitis}

\section{Areas of Consensus}

Patients with alcoholic hepatitis and MELD $<20$ or $\mathrm{DF}<32$ have a mortality rate of $10 \%$ at 90 days and, therefore, cannot be considered as non-severe. It is proposed to use the term "moderate alcoholic hepatitis" for patients with alcoholic hepatitis and MELD 11-20; and mild alcoholic hepatitis with MELD $\leq 10$.

The three-month natural history of moderate alcoholic hepatitis (MELD score 11-20) needs to be defined. It is also necessary to define whether pharmacological therapy is required for patients with moderate alcoholic hepatitis.

A team approach with hepatologist, addiction specialist, nutritional expert and social work is desirable for treatment of patients with severe $\mathrm{AH}$.

Liver biopsy is advised prior to treatment for severe alcoholic hepatitis but is not mandatory. Inpatient management of severe $\mathrm{AH}$ includes:

- Nutritional Assessment and Optimal Replacement of protein and calories.

- Transfer to the Intensive Care Unit for organ support.

- Meticulous investigation for infection. When infection is diagnosed or strongly suspected, broad spectrum antibiotics should be started within one hour.

- Steroids therapy if there are no contraindications.

- Lille score should be the preferred tool to access response to steroid therapy.

- Liver transplantation should be offered to highly selected patients who fail medical management.

Steroid therapy reduces one-month mortality but does not impact medium term mortality. The absence of impact on medium term survival emphasizes the need for newer therapies.

Living donor liver transplantation should be carried out only after approval of local ethics committees and in centers with large experience and where deceased donor transplantation is not available.

\begin{tabular}{|l|}
\hline \multicolumn{1}{|c|}{ Unmet Needs and Opportunities for Future Study } \\
\hline \multicolumn{1}{|c|}{} \\
\hline Validation of histological endpoints as a surrogate for a clinical event. \\
\hline $\begin{array}{l}\text { Non-invasive methods for the diagnosis of alcoholic hepatitis when considering the limitations of liver } \\
\text { biopsy. }\end{array}$ \\
\hline $\begin{array}{l}\text { Studies comparing long-term alcoholism behavior of patients with early transplantation to patients } \\
\text { undergoing transplantation after abstaining from alcohol for a 6-month period. }\end{array}$ \\
\hline \multicolumn{2}{|c|}{} \\
\hline Routine use of antibiotics in all patients with severe alcoholic hepatitis. \\
\hline Specific contraindications to steroids. \\
\hline Criteria for "futility" to exclude patients from studies. \\
\hline
\end{tabular}




\section{Table 3. Next Steps for Future Trials}

\section{Areas of Consensus}

The optimal time frame of survival endpoint for studies testing new molecules in the setting of severe alcoholic hepatitis should be 90 days.

Liver biopsy is recommended for patients entering Phase I-II studies and may be informative for Phase III studies.

Calculation of sample size in future Phase III studies without liver biopsy should integrate the risk of misclassification based on NIAAA classification as also inter-observer agreement in liver biopsy interpretation

Use of NIAAA classification of alcoholic hepatitis is recommended for design of studies without liver biopsy. Only patients with probable or definite $\mathrm{AH}$ should be candidates in future studies testing new drugs.

Lille and other scores should be recorded at different time points to determine response to treatments such as regenerative therapies.

There is the need for surrogate endpoints for survival. We propose a combined endpoint of survival and decrease in MELD score as an endpoint for efficacy of a treatment. That is, either mortality or patient who is alive but without a decrease in MELD score is study drug failure.

\section{Unmet Needs and Opportunities for Future Study}

Validation of histological endpoints as a surrogate for a clinical event.

Developing specific stage/fibrosis scoring systems for ALD

The concordance between the clinic syndrome of alcoholic hepatitis and histological lesion of steatohepatitis needs formal definition.

The impact of sarcopenia and frailty on outcome and selection of treatment should be explored.

Criteria of drug-induced liver injury (DILI) and drug induced kidney injury adapted to patients with severe alcoholic hepatitis should be established.

Markers of futility for pharmacological therapy are required to exclude patients from clinical trials (example MELD score $>35$ which is associated with $80 \%$ risk of 90 -day mortality).

Consensus for endpoints for Phase I and II studies. 


\section{Table 4. Translational Science and Evolving Biomarkers}

Areas of Consensus

Translational science can aid the diagnosis and management of alcoholic hepatitis by developing biomarkers to:

- $\quad$ Predict disease outcomes

- Predict response to therapy

- Detect infection early

- Predict steroid resistance

- Identify drug resistance

- Detect alcohol use recidivism

Clinical criteria to be used in the diagnosis of ALD and AH in biomarker studies should be defined by:

- Biopsy proven ALD or AH

- Clinical diagnosis

- MELD score to determine severity of disease

Recommended control patient groups:

- Chronic alcoholics without liver disease

- Age \& sex matched healthy controls

- Patients with cirrhosis

- Patients with other liver disease

Large scale studies in large populations should take advantage of "omics" technologies in biomarker discovery.

Genetic markers should be used in evaluation, prognosis and management of $\mathrm{AH}$.

Animal models should be used to assess potential biomarkers to establish proof-of-concept for human studies.

\section{Unmet Needs and Opportunities for Future Study}

Animal models that accurately model human disease are needed where cholestasis, inflammation, and fibrosis are present together.:

- Alcohol associated liver fibrosis (current models for ALD do not exhibit significant fibrosis)

- Alcoholic hepatitis

- Alcoholic cirrhosis

Need for new biomarkers to:

- Assess ongoing alcohol use in AH patients after hospital discharge

- Assess inflammation (Cytokines, Chemokines, PAMPs/DAMPs)

- Differentiate between infection mediated inflammation and sterile inflammation

- Predict organ failure

- Assess liver regeneration 